\title{
Toward the Toxicology of Some Nitro-Compounds
}

\author{
Bisheng Tan ${ }^{1^{*}}$, Longyu Liao ${ }^{1}$, Yang Zhou ${ }^{1}$, Xinping Long ${ }^{2 *}$, Jinshan Li $^{1}$ \\ ${ }^{1}$ Institute of Chemical Materials, China Academy of Engineering Physics (CAEP), Mianyang, P. R. China \\ 621900; 를 Academy of Engineering Physics (CAEP), Mianyang, P. R. China 621900. \\ Email: tanbs_my@caep.cn
}

\begin{abstract}
Nitro-compounds are widely used in medicine, agriculture and industry. Their toxicity issues attract more and more attention. There are a lot of factors influncing toxicity, and the degrees are different from one another. Some physical properties of compounds, such as the surface properties, may play crucial roles in the toxicity of these compounds. Twenty seven parameters of twenty eight nitro-compounds, including molecular size related parameters, molecular surface electrostatic potential based parameters, solubility parameters, molecular cohesive energies, surface tensions, dielectric constants and Mulliken charges of nitro groups, were considered to correlate with toxicity (semi-lethal dose, $L D_{50}$ ) of the nitro-compounds. Fourteen parameters with higher correlation coefficients were selected to join in the modelling process of heredity and variation (genetic function approximation), further screening toxicity related parameters, the screened parameters are molecular surface electrostatic potentials based $\sigma_{+}^{2}, \sigma_{-}^{2}, v \sigma_{t o t}^{2}, A_{S}^{+}, \bar{V}_{S}, \log P_{\mathrm{OW}}$, and nitro group's Mulliken charges $q_{\mathrm{NO}_{2}}$. Building the linear relation between toxicity and these screened parameters, and quantitatively studying the toxicity of nitro-compounds, this work may help us evaluate health risks and approach the toxicology of nitro-compouds.
\end{abstract}

Keywords: Acute toxicity test; semi-lethal dose; electrostatic potentials; genetic function approximation; toxicology

\section{Introduction}

Nitroaromatic compounds are widely used in medicine, industry and agriculture. Nitroaromatic pesticides and explosive residues are considered as being toxic environmental pollutants [1]. Therefore, it is very important to study the toxicity of nitro containing compounds.

The toxicity studies cover a wide field, ranging from carcinogenesis, the whole body system (reproductive system, immune system, nervous system, digestive system, respiratory system, cardiovascular system, dermal system, endocrine system, the individual visceras (liver, kidney, belly, et al.), to acute toxicity test.

Acute toxicity test, also called single dose acute toxicity test, is a kind of toxic reaction of the animal subject one or many times tests per $24 \mathrm{hr}$ (6-8hr interval between two tests), including the general behaviours, the changes of appearance, the changes of gross morphology and death effects, which is characterized half lethal dose $\left(L D_{50}\right.$, in uinit of $\left.\mathrm{mg} / \mathrm{kg}\right)$. The higher is the $L D_{50}$, the lower is the toxicity.

The toxicology of nitro-compounds is not well known so far, some investigators argued that covalent addition reactions between metabolic intermediates of nitro-compounds and cell proteins is the mechanism of toxication $[2 \sim 4]$.

The cytotoxic, mutagenic and carcinogenic properties of nitroaromatic environmental pollutants are frequently related to their electron-accepting properties [1,5]. Chung et al explored the influences of nitro group on genic mutation and toxicity [6].

In the past few years, quantitative structure-activity relationship (QSAR) [7 9] plays more and more important role in the study of the relationship between the molecular structures of chemicals and their bioactivities, which can be used to quickly predict biotoxicities of newly found or synthesized compounds.

Hansch et al. proposed Hansch-Fujita model and turned a new page of QSAR or QSPR [10,11] in the research of activities of compounds. By the beginning of twentieth century, it is commonly believed that bioactivities of compounds are mainly determined by their physical properties, including vapor pressure, 
Henry' s law constants, water solubility, octanol/water partition coefficients, heats of formation and ionization potentials of explosive TNT [12], acidity and basicity of hydrogenbond, solubilit, surface tension, dipole moment, refractive index and dielectric constant [13], solid, liquid and gaseous enthalpies of formation in terms molecular electrostatic potentials [14], density, vaporization enthalpy, heat capacity, surface tension, isothermal compressibility and dielectric constant [15 18], the role of polarization in the interaction between chemistry and biology [19]. Nano toxicology also begins to attract more and more attention with the fast development of nano technology in recent years [20].

In this work the correlation between toxicity of 28 kinds of nitro compounds and their properties were studied, which include molecular volumn, superficial area, enthalpy of vaporization, density of cohesive energy, solubility parameter, polarizability, dipole moment, dielectric constant, surface tension, charge distribution of nitro groups, molecular surface electrostatic potential based functions, and solubility parameters, surface tension, dipole moment and molecular surface electrostatic potentials based parameters were screened to build the relationship with $L D_{50}^{\exp }$.

\section{Theory}

Molecular electrostatic potential and related equations proposed by Politzer [21] were displayed in formulas (1) to (8):

$$
V(\mathrm{r})=\sum_{A} \frac{Z_{A}}{\left|\mathrm{R}_{\mathrm{A}}-\mathrm{r}\right|}-\int \frac{\rho\left(\mathrm{r}^{\prime}\right) \mathrm{dr} \mathrm{r}^{\prime}}{\left|\mathrm{r}^{\prime}-\mathrm{r}\right|}
$$

where $V(\mathrm{r}), Z_{A}$ and $\rho(\mathrm{r})$ denote electrostatic potential, charge on $A$ nuclus located at $R_{\mathrm{A}}$ and electron density, respectively. The relative properties are defined as $\mathrm{P}=f\left[A_{S}, A_{S}^{+}, A_{S}^{-}, \bar{V}_{S}^{+}, \bar{V}_{S}^{-}, \bar{V}_{S}\right.$, $\left.\Pi, \sigma_{+}^{2}, \sigma_{-}^{2}, \sigma_{t o t}^{2}, v, v \sigma_{\text {tot }}^{2}, V_{\mathrm{S}, \min }, V_{\mathrm{S}, \max }\right]$, where $A_{S}, A_{S}^{+}$and $A_{S}^{-}$are molecular total surface area, electropositive surface area and electronegative surface area, respectively. $V_{\mathrm{S}, \min } V_{\mathrm{S} \text {,max }}$ and $\Pi$ are the minimum surface electrostatic potential, maximum surface electrostatic potential and average deviation of surface electrostatic potential at $r$ point, respectively [22].

$$
\begin{gathered}
\sigma_{\text {tot }}^{2}=\sigma_{+}^{2}+\sigma_{-}^{2}=\frac{1}{r} \sum_{i=1}^{r}\left[V_{S}^{+}\left(\mathrm{r}_{i}\right)-\bar{V}_{S}^{+}\right]^{2}+\frac{1}{s} \sum_{j=1}^{s}\left[V_{S}^{-}\left(\mathrm{r}_{j}\right)-\bar{V}_{S}^{-}\right]^{2} \\
\bar{V}_{S}^{+}\left(\mathrm{r}_{i}\right)=\frac{1}{r} \sum_{i=1}^{r} V_{S}^{+}\left(\mathrm{r}_{i}\right), \bar{V}_{S}^{-}=\frac{1}{s} \sum_{j=1}^{s} V_{S}^{-}\left(\mathrm{r}_{j}\right)
\end{gathered}
$$

where $\sigma_{+}^{2}$ and $\sigma_{-}^{2}$ are positive and negative standard deviation of molecular electrostatic potential respectively, $\sigma_{\text {tot }}^{2}=\sigma_{+}^{2}+\sigma_{-}^{2} . V_{S}^{+}\left(\mathrm{r}_{i}\right)$ and $V_{S}^{-}\left(\mathrm{r}_{j}\right)$ are positive and negative electrostatic potential located at $\mathrm{r}_{i}$ and $\mathrm{r}_{j}$, respectively. $\Pi$ is the average deviation of $V(\mathrm{r})$ on the molecular surface, interpreted as the local polarity, or internal charge separation.

$$
\begin{gathered}
v=\frac{\sigma_{+}^{2} \sigma_{-}^{2}}{\left[\sigma_{\mathrm{tot}}^{2}\right]^{2}} \\
\Pi=\frac{1}{2} \sum_{i=1}^{n}\left|V\left(r_{\mathrm{i}}\right)-\bar{V}_{\mathrm{S}}\right|
\end{gathered}
$$

Enthalpy of vaporization, coefficient of diffusion and water/oil partition coefficient [23 25] are derived from molecular surface electrostatic potentials, expressed as formulas ( 6) (8).

$$
\begin{gathered}
\Delta_{\text {vap }} H^{\circ}=1.355 A_{\mathrm{S}}^{0.5}+1.176\left(v \sigma_{\text {tot }}^{2}\right)^{0.5}-10.433 \\
D \times 10^{7}\left(\mathrm{~cm}^{2} / \mathrm{s}\right)=533.5(A s)^{-1}-0.03168 \sigma_{+}^{2}+0.01425 \sigma_{-}^{2}-1.620 \\
\log P_{\text {ow }}=0.03 A s-0.00472 N \sigma_{-}^{2}-0.000963 A s \Pi-0.504 \\
N=n_{\mathrm{N}}+2 n_{\mathrm{O}}
\end{gathered}
$$

The cohesive energy $E_{\text {coh }}[26]$ of a substance in a condensed state is defined as the increase in internal energy $U$ (in $\mathrm{J} / \mathrm{mol}$ ) per mole of substance if all the intermolecular forces are eliminated: 


$$
\begin{gathered}
\text { The cohesive energy } \equiv E_{\text {coh }}=\Delta U \\
\text { Solubility parameter } \delta=\left(\frac{E_{\text {coh }}}{V}\right)^{0.5}=\left(\frac{\Delta_{\text {vap }} H-R T}{V}\right)^{0.5}
\end{gathered}
$$

where $V$ is van der Waals volume of a specific molecule.

Molecular polarizability proposed by Hansch [27] is defined as Equ. (11):

$$
\mathrm{a}(0)\left[\AA^{3} / \text { molecule }\right]=0.27( \pm 0.011) \mathrm{NVE}
$$

where $\alpha(0)$ and $N V E$ are volume of polarization and molecular total valence electrons, respectively. The relationship between surface tension and solubility parameter [28] of a compound can be expressed as Equ. (12):

$$
\gamma=a \delta^{2} V_{m}^{0.45}+b
$$

where empirical parameter $a$ and $b$ are 0.0145 and -24.283 , obtained from linear fitting method.

Dielectric constants $\left(\varepsilon^{\mathrm{cal}}\right)$ of the compounds are obtained from the fitting method in terms of electrostatic potential parameters and solubility parameters.

$$
\varepsilon^{c a l}=f\left(\delta, \delta^{2} \sigma_{t o t}^{0.4}\right)
$$

Mulliken charges of nitro groups were computed according to Equ. (14).

$$
q_{\mathrm{NO}_{2}}=q_{N}+q_{\mathrm{O}(1)}+q_{\mathrm{O}(2)}
$$

\section{Computational Details}

Molecular structure optimization were carried out by hybrid density function theory B3LYP/6-31G(d,p) [29,30], molecular van der Waals volumes, surface areas and surface electrostatic potentials were calculated by multifunctional wave function code Multiwfn [31]. Surface tensions and slubility parameters were fitted with the combined parameter $\delta^{2} V_{m}^{0.45}$ in terms of the literature method [28], the fitted squared- $R$ (correlation coefficient) reaches 0.9803 , which were displayed in Table 1.

Table 1. The calculated electrostatic potential based parameters $\left(\sigma_{t o t}^{2}, \sigma_{+}^{2}, \sigma_{-}^{2}, v, \Delta_{\text {vap }} H^{\circ}\right)$, solubility parameters $(\delta)$, volumns $\left(V_{\mathrm{m}}\right)$, molecular areas $\left(A_{\mathrm{s}}\right)$, cohesive energies $\left(E_{\mathrm{coh}}\right)$ and the predicted surface tensions $(\gamma)$.

\begin{tabular}{llllllllllll}
\hline & $V_{\mathrm{m}}$ & $A_{\mathrm{s}}$ & $\sigma_{\text {tot }}^{2}$ & $\sigma_{+}^{2}$ & $\sigma_{-}^{2}$ & $v$ & $\Delta_{\text {vap }} H^{\circ}$ & $E_{\text {coh } \times 10^{-4}} \delta$ & $\delta^{2} V_{m}^{0.45}$ & $\gamma$ \\
\hline Et & 39.300 & 83.755 & 3.592 & 3.156 & 0.441 & 0.1079 & 2.700 & 1.378 & 18.722 & 1828.863 & 1.034 \\
Propane & 53.669 & 105.309 & 3.409 & 2.844 & 0.565 & 0.1383 & 4.279 & 2.038 & 19.488 & 2279.870 & 7.571 \\
Butane & 67.601 & 126.571 & 3.146 & 2.589 & 0.558 & 0.1460 & 5.608 & 2.594 & 19.590 & 2555.923 & 12.530 \\
Benzene & 69.486 & 125.463 & 36.835 & 10.041 & 26.794 & 0.1983 & 7.923 & 3.563 & 22.644 & 3457.483 & 28.800 \\
BA & 78.725 & 139.236 & 147.932 & 101.752 & 46.180 & 0.2147 & 12.183 & 5.345 & 26.057 & 4842.833 & 44.880 \\
BP & 75.077 & 134.099 & 151.855 & 112.261 & 39.594 & 0.1928 & 11.621 & 5.110 & 26.089 & 4752.181 & 40.900 \\
MeB & 83.468 & 146.282 & 38.282 & 8.291 & 29.991 & 0.1697 & 8.953 & 3.994 & 21.874 & 3503.809 & 28.540 \\
PD & 65.709 & 120.416 & 127.074 & 24.746 & 102.328 & 0.1568 & 9.685 & 4.300 & 25.582 & 4303.286 & 37.120 \\
NB & 86.085 & 150.292 & 155.002 & 48.976 & 106.026 & 0.2161 & 12.985 & 5.185 & 24.542 & 4472.365 & 43.900 \\
\hline
\end{tabular}

Note: Parameters $V_{\mathrm{m}}, A_{\mathrm{s}}, \sigma_{\text {tot }}^{2}, \sigma_{+}^{2}, \sigma_{-}^{2}, \Delta_{\text {vap }} H^{\circ}, E_{\text {coh }}, \delta, \gamma$ are in unit of $\mathrm{cm}^{3} / \mathrm{mol}, \AA^{2},(\mathrm{kcal} / \mathrm{mol})^{2},(\mathrm{kcal} / \mathrm{mol})^{2}$, $\left.(\mathrm{kcal} / \mathrm{mol})^{2}, \mathrm{kcal} / \mathrm{mol}, \mathrm{J} / \mathrm{mol}, \mathrm{J} / \mathrm{cm}^{3}\right)^{1 / 2}, \mathrm{mN} / \mathrm{m}$, respectively.

The experimental surface tensions in Table 1 were taken from the data of $20^{\circ} \mathrm{C}[32]$. Dielectric constants of these compounds were calculated according to the fitted genetic function approximation method, and the fitted equation was expressed as follows:

$\varepsilon^{\text {cal }}=-3.861^{*} \mathrm{X} 1-14.859^{*} \hat{R}(23.757-\mathrm{X} 1)+33.657^{*} \hat{R}(23.316-\mathrm{X} 1)-0.034^{*} \hat{R}(5492.795-\mathrm{X} 2)+163.068, \mathrm{X} 1=\delta$, $\mathrm{X} 2=\delta^{2} \sigma_{\text {tot }}^{0.4}$.

The experimental dielectric constants [32] were listed in Table 2, and the square of the fitted linear correlation coefficient reaches 0.9979 . 
$\varepsilon^{\mathrm{cal}}=-3.861^{*} \mathrm{X}_{1}-14.859^{*} \hat{R}\left(23.757-\mathrm{X}_{1}\right)+33.657^{*} \hat{R}\left(23.316-\mathrm{X}_{1}\right)-0.034^{*} \hat{R}\left(5492.795-\mathrm{X}_{2}\right)+163.068, \quad \mathrm{X}_{1}=\delta$, $\mathrm{X}_{2}=\delta^{2} \sigma_{\text {tot }}^{0.4}$.

Table 2. The calculated electrostatic potential based parameters, solubility parameters, moleculear volumns, cohesive energies, the number of valence electrons, dipole moments, Mulliken charges of nitro groups and the predicted dielectric constants.

\begin{tabular}{llllllllll}
\hline & $\mathrm{m}-\mathrm{DNB}$ & $\mathrm{m}-\mathrm{NTul}$ & $\mathrm{NB}$ & $\mathrm{NEt}$ & $\mathrm{NMe}$ & o-NTul & p-NBA & p-NTul & TNT \\
\hline$V_{\mathrm{m}}$ & 102.942 & 99.791 & 86.085 & 56.530 & 42.563 & 99.085 & 95.339 & 99.864 & 132.754 \\
$A_{\mathrm{s}}$ & 175.830 & 171.182 & 150.292 & 110.544 & 89.812 & 167.152 & 163.927 & 171.128 & 216.994 \\
$\sigma_{\text {tot }}^{2}$ & 96.386 & 158.003 & 155.002 & 101.578 & 107.300 & 158.479 & 361.630 & 165.486 & 91.350 \\
$\sigma_{+}^{2}$ & 49.823 & 33.540 & 48.976 & 35.642 & 47.327 & 35.360 & 188.916 & 35.673 & 61.362 \\
$\sigma_{-}^{2}$ & 46.563 & 124.463 & 106.026 & 65.936 & 59.973 & 103.119 & 172.714 & 129.813 & 29.989 \\
$\nu^{2}$ & 0.2497 & 0.1672 & 0.2161 & 0.2278 & 0.2465 & 0.1452 & 0.2495 & 0.1691 & 0.2205 \\
$\Delta_{\text {vap }} H^{\circ}$ & 13.304 & 13.340 & 12.985 & 9.470 & 8.456 & 12.727 & 18.086 & 13.514 & 14.805 \\
$E_{\text {coh } \times 10^{-4}}$ & 5.318 & 5.334 & 5.185 & 3.714 & 3.290 & 5.077 & 7.319 & 5.406 & 5.946 \\
$\delta$ & 22.730 & 23.119 & 24.542 & 25.633 & 27.803 & 22.636 & 27.708 & 23.267 & 21.164 \\
$\mathrm{NVE}$ & 62 & 52 & 46 & 30 & 24 & 52 & 52 & 52 & 84 \\
$\alpha(0)$ & 16.74 & 14.04 & 12.42 & 8.10 & 6.48 & 14.04 & 14.04 & 14.04 & 22.68 \\
$\mu$ & 4.198 & 4.889 & 4.557 & 3.599 & 3.488 & 4.304 & 7.577 & 5.237 & 1.546 \\
$\varepsilon$ & 2.8 & 23.8 & 35.7 & 19.7 & 39.4 & 27.4 & 56.3 & 22.2 & 22.0 \\
$q_{\mathrm{NO}_{2}}$ & -0.372 & -0.410 & -0.406 & -0.310 & -0.303 & -0.425 & -0.462 & -0.417 & -0.355 \\
\hline
\end{tabular}

Note: $V_{\mathrm{m}}, A_{\mathrm{s}}, \sigma_{\text {tot }}^{2}, \sigma_{+}^{2}, \sigma_{-}^{2}, \Delta_{\text {vap }} H^{\circ}, E_{\mathrm{coh}}, \delta, \alpha(0), \mu, \varepsilon, q_{\mathrm{NO}_{2}}$ are in unit of cm $3 / \mathrm{mol}, \AA^{2},(\mathrm{kcal} / \mathrm{mol})^{2},(\mathrm{kcal} / \mathrm{mol})$ ${ }^{2},(\mathrm{kcal} / \mathrm{mol})^{2}, \mathrm{kcal} / \mathrm{mol}, \mathrm{J} / \mathrm{mol},\left(\mathrm{J} / \mathrm{cm}^{3}\right)^{1 / 2}, \AA^{3}$, Debye, a.u., respectively.

The molecular electrostatic potential related parameters derived from Equs. (1) (8) were carried out by Multiwfn code [31]. The correlation between $L D_{50}^{\exp }$ and these parameters was computed by Equ. (15).

$$
\Omega_{x, y}=\frac{\operatorname{Cov}(\mathrm{X}, \mathrm{Y})}{\sigma_{x} \sigma_{y}}
$$

where $\Omega_{x, y}, \operatorname{Cov}(\mathrm{X}, \mathrm{Y}), \sigma_{x}$ and $\sigma_{y}$ are correlation coefficient, covariance, standard deviation of array $x$ and array $y$, respectively.

All of these parameters, those with low correlation coefficient $(<0.3)$ were eliminated, the remained ones including molecular electrostatic potential related parameters $\sigma_{\text {tot }}^{2}, \sigma_{+}^{2}, \sigma_{-}^{2}, V_{\mathrm{S}, \min }, A_{S}^{+}, \bar{V}_{S}, v \sigma_{t o t}^{2}$, Log $P_{\text {OW }}$, solubility parameter $\delta$, dipole moment $\mu$, dielectic constant $\varepsilon$, surface tension $\gamma$, combining function $\delta^{2} V_{m}^{0.45}$ and Mulliken charge of nitro groups were employed to join the genetic function approximation modelling, seven parameters were preserved in the $L D_{50}^{\text {cal }}$ related model, including $\sigma_{+}^{2}$, $\sigma_{-}^{2}, q_{\mathrm{NO}_{2}}, A_{S}^{+}, \bar{V}_{S}, v \sigma_{\text {tot }}^{2}, \log P_{\mathrm{OW}}$.

Genetic algorithm (GA) is a compiled computational program that simulates natural evolution of heredity, mutation, selection and crossover of active organismals [33].

\section{Results and Discussion}

A series of physical parameters in terms of formula in theoretical and computational part of 28 nitrocompounds were obtained and displayed in Table 3, and the correlation coefficients between $L D_{50}^{\exp }$ and the calculated parameters were listed at the end of Table 3 to Table 6 . 
Table 3. The correlation prediction between $L D_{50}^{\exp }[34]$ and 6 parameters.

\begin{tabular}{|c|c|c|c|c|c|c|c|}
\hline & $L D_{50}^{\exp }$ & $V_{\mathrm{m}}$ & $\sigma_{t o t}^{2}$ & $\sigma_{+}^{2}$ & $\sigma_{-}^{2}$ & $v$ & $\Delta_{\text {vap }} H^{\circ}$ \\
\hline 1-Cl-2-NB & 0.27 & 98.169 & 150.470 & 56.752 & 93.718 & 0.2349 & 14.009 \\
\hline 1-Cl-4-NB & 0.81 & 98.723 & 138.575 & 44.362 & 94.213 & 0.2176 & 13.636 \\
\hline 23-DNTul & 1.12 & 116.538 & 103.441 & 49.110 & 54.330 & 0.2494 & 14.324 \\
\hline 24-DNBP & 0.035 & 107.659 & 101.031 & 49.080 & 51.951 & 0.2498 & 13.750 \\
\hline 24-DNTul & 0.27 & 115.928 & 90.376 & 38.501 & 51.874 & 0.2445 & 13.911 \\
\hline 25-DNTul & 0.71 & 115.882 & 75.370 & 29.208 & 46.162 & 0.2374 & 13.356 \\
\hline 26-DNTul & 0.18 & 115.462 & 94.799 & 49.013 & 45.786 & 0.2497 & 13.980 \\
\hline $2-\mathrm{NI}$ & $0.08^{\mathrm{a}}$ & 70.698 & 271.968 & 169.513 & 102.456 & 0.2348 & 14.466 \\
\hline 34-DNTul & 1.07 & 116.849 & 108.690 & 49.041 & 59.649 & 0.2476 & 14.649 \\
\hline TNBP & $0.6^{\mathrm{c}}$ & 124.621 & 134.646 & 96.680 & 37.966 & 0.2025 & 15.205 \\
\hline$m$-DNB & $0.01^{\mathrm{c}}$ & 102.942 & 96.386 & 49.823 & 46.563 & 0.2497 & 13.304 \\
\hline$m$-NBA & 0.54 & 95.209 & 278.126 & 150.506 & 127.620 & 0.2483 & 16.693 \\
\hline$m$-NTul & 1.07 & 99.791 & 158.003 & 33.540 & 124.463 & 0.1672 & 13.340 \\
\hline NB & $0.75-1.0^{c}$ & 86.085 & 155.002 & 48.976 & 106.026 & 0.2161 & 12.985 \\
\hline $\mathrm{NBu}$ & $0.50-0.75^{\mathrm{b}}$ & 84.602 & 95.743 & 26.386 & 69.357 & 0.1996 & 11.507 \\
\hline NEt & 1.10 & 56.530 & 101.578 & 35.642 & 65.936 & 0.2278 & 9.470 \\
\hline $\mathrm{NMe}$ & $1.44^{\mathrm{a}}$ & 42.563 & 107.300 & 47.327 & 59.973 & 0.2465 & 8.456 \\
\hline NPr & $0.50-0.75^{\mathrm{b}}$ & 70.383 & 93.627 & 25.681 & 67.945 & 0.1990 & 10.231 \\
\hline$o-\mathrm{NBA}$ & 3.56 & 94.143 & 235.101 & 105.549 & 129.552 & 0.2474 & 15.711 \\
\hline$o-\mathrm{NBP}$ & 2.828 & 91.571 & 311.656 & 160.509 & 151.147 & 0.2498 & 16.968 \\
\hline$o-\mathrm{NTul}$ & 0.89 & 99.085 & 158.479 & 35.360 & 103.119 & 0.1452 & 12.727 \\
\hline$p$-DNB & $0.0294^{\mathrm{d}}$ & 102.687 & 82.999 & 39.628 & 43.371 & 0.2495 & 12.874 \\
\hline$p$-NBA & 3.25 & 95.339 & 361.630 & 188.916 & 172.714 & 0.2495 & 18.086 \\
\hline$p$-NBP & 0.616 & 92.006 & 297.564 & 182.109 & 115.455 & 0.2374 & 16.536 \\
\hline$p$-NTul & 2.14 & 99.864 & 165.486 & 35.673 & 129.813 & 0.1691 & 13.514 \\
\hline Tetryl & $0.5^{\mathrm{c}}$ & 159.537 & 144.080 & 109.412 & 34.668 & 0.1827 & 17.118 \\
\hline TNT & $0.48^{\mathrm{d}}$ & 132.754 & 91.350 & 61.362 & 29.989 & 0.2205 & 14.805 \\
\hline LLM-105 & 2.000 & 118.130 & 187.285 & 109.731 & 77.554 & 02426 & 16.486 \\
\hline Corr. & 1.000 & -0.1852 & 0.5623 & 0.3569 & 0.6814 & 0.0439 & 0.2911 \\
\hline
\end{tabular}

Note: $L D_{50}^{\exp }, V_{\mathrm{m}}, A_{\mathrm{s}}, \sigma_{\text {tot }}^{2}, \sigma_{+}^{2}, \sigma_{-}^{2}, \Delta_{\text {vap }} H^{\circ}$ are in unit of $\mathrm{g} / \mathrm{kg}, \mathrm{cm}^{3} / \mathrm{mol}, \AA^{2},(\mathrm{kcal} / \mathrm{mol})^{2},(\mathrm{kcal} / \mathrm{mol})^{2}$, $(\mathrm{kcal} / \mathrm{mol})^{2}, \mathrm{kcal} / \mathrm{mol}$, respectively. a, b, c,d denote, mouse, rabbit, dog and cat, respectively.

Table 4.8 parameters in relation to $L D_{50}^{\exp }$.

\begin{tabular}{lllllllll}
\hline & $E_{\text {coh }} \times 10^{-4}$ & $\delta$ & NVE & $\alpha(0)$ & $\mu$ & $\varepsilon$ & $\delta^{2} V_{m}^{0.45}$ & $\gamma$ \\
\hline 1-Cl-2-NB & 5.613 & 23.913 & 52 & 14.04 & 5.032 & 28.79 & 4504.604 & 41.034 \\
1-Cl-4-NB & 5.457 & 23.512 & 52 & 14.04 & 2.922 & 17.45 & 4365.836 & 39.022 \\
23-DNTul & 5.745 & 22.204 & 68 & 18.36 & 6.586 & 12.82 & 4195.395 & 36.550 \\
24-DNBP & 5.505 & 22.613 & 68 & 18.36 & 3.378 & 6.47 & 4198.934 & 36.602 \\
24-DNTul & 5.572 & 21.924 & 68 & 18.36 & 4.843 & 11.05 & 4080.603 & 34.886 \\
25-DNTul & 5.340 & 21.467 & 68 & 18.36 & 0.885 & 10.76 & 3911.559 & 32.435 \\
26-DNTul & 5.601 & 22.026 & 68 & 18.36 & 2.956 & 11.57 & 4111.202 & 35.329 \\
2-NI & 5.805 & 28.654 & 42 & 11.34 & 5.544 & 52.42 & 5579.608 & 56.621 \\
34-DNTul & 5.881 & 22.435 & 68 & 18.36 & 7.308 & 11.98 & 4288.283 & 37.897 \\
TNBP & 6.114 & 22.149 & 84 & 22.68 & 1.786 & 25.30 & 4302.534 & 38.104 \\
$m$-DNB & 5.318 & 22.730 & 62 & 16.74 & 4.198 & 2.80 & 4157.819 & 36.005 \\
$m$-NBA & 6.736 & 26.600 & 52 & 14.04 & 5.996 & 60.35 & 5497.54 & 55.431 \\
$m$-NTul & 5.334 & 23.119 & 52 & 14.04 & 4.889 & 23.80 & 4241.595 & 37.220 \\
NB & 5.185 & 24.542 & 46 & 12.42 & 4.557 & 35.70 & 4472.365 & 43.900
\end{tabular}




\begin{tabular}{lllllllll}
$\mathrm{NBu}$ & 4.567 & 23.233 & 42 & 11.34 & 3.855 & 3.965 & 3976.783 & 33.380 \\
$\mathrm{NEt}$ & 3.714 & 25.633 & 30 & 8.10 & 3.599 & 19.70 & 4037.61 & 34.262 \\
$\mathrm{NMe}$ & 3.290 & 27.803 & 24 & 6.48 & 3.488 & 39.40 & 4180.689 & 36.337 \\
$\mathrm{NPr}$ & 4.033 & 23.937 & 36 & 9.72 & 3.757 & 4.18 & 3885.974 & 32.064 \\
$o$-NBA & 6.326 & 25.921 & 52 & 14.04 & 4.726 & 62.98 & 5194.074 & 51.031 \\
o-NBP & 6.852 & 27.354 & 52 & 14.04 & 5.822 & 57.44 & 5712.586 & 58.550 \\
o-NTul & 5.077 & 22.636 & 52 & 14.04 & 4.304 & 27.40 & 4053.246 & 34.489 \\
$p$-DNB & 5.139 & 22.370 & 62 & 16.74 & 0 & 1.56 & 4022.666 & 34.046 \\
$p$-NBA & 7.319 & 27.708 & 52 & 14.04 & 7.577 & 56.30 & 5968.733 & 62.264 \\
$p$-NBP & 6.671 & 26.926 & 52 & 14.04 & 5.341 & 59.10 & 5547.036 & 56.149 \\
$p$-NTul & 5.406 & 23.267 & 52 & 14.04 & 5.237 & 22.20 & 4297.489 & 38.031 \\
Tetryl & 6.914 & 20.818 & 106 & 28.62 & 3.352 & 44.61 & 4247.816 & 37.310 \\
TNT & 5.946 & 21.164 & 84 & 22.68 & 1.546 & 22.00 & 4041.727 & 34.322 \\
LLM-105 & 7.145 & 24.594 & 80 & 21.60 & 5.402 & 48.295 & 4905.232 & 46.843 \\
Corr. & $\mathbf{0 . 2 9 1 2}$ & $\mathbf{0 . 4 5 6 6}$ & $\mathbf{- 0 . 2 3 4 6}$ & $\mathbf{- 0 . 2 3 4 6}$ & $\mathbf{0 . 4 4 1 1}$ & $\mathbf{0 . 5 4 7 3}$ & $\mathbf{0 . 5 1 0 0}$ & $\mathbf{0 . 5 0 6 0}$ \\
\hline
\end{tabular}

Note: $E_{\text {coh }}, \delta, \alpha(0), \mu, \gamma, q_{\mathrm{NO}_{2}}$ are in unit of $\mathrm{J} / \mathrm{mol},\left(\mathrm{J} / \mathrm{cm}^{3}\right)^{1 / 2}, \AA^{3}$, Debye, dyne/cm, a.u., respectively.

Table 5. 7 electrostatic potential parameters in relation to $L D_{50}^{\exp }$.

\begin{tabular}{|c|c|c|c|c|c|c|c|c|}
\hline & $L D_{50}$ & $V_{\mathrm{S}, \text { min }}$ & $V_{\mathrm{S}, \max }$ & $A_{S}$ & $A_{S}^{+}$ & $A_{S}^{-}$ & $\bar{V}_{S}$ & $\bar{V}_{S}^{+}$ \\
\hline 1-Cl-2-NB & 0.27 & -32.190 & 26.761 & 165.851 & 103.118 & 62.733 & 1.809 & 12.018 \\
\hline 1-Cl-4-NB & 0.81 & -30.247 & 25.312 & 168.939 & 111.684 & 57.256 & 2.936 & 10.969 \\
\hline 23-DNTul & 1.12 & -30.132 & 30.378 & 192.176 & 116.302 & 75.874 & 2.063 & 15.414 \\
\hline 24-DNBP & 0.035 & -28.017 & 35.737 & 181.908 & 109.672 & 72.235 & 4.239 & 15.591 \\
\hline 24-DNTul & 0.27 & -27.936 & 30.561 & 192.820 & 120.242 & 72.578 & 3.801 & 15.381 \\
\hline 25-DNTul & 0.71 & -26.020 & 26.231 & 192.792 & 122.262 & 70.530 & 4.030 & 14.540 \\
\hline 26-DNTul & 0.18 & -25.924 & 29.404 & 190.292 & 117.537 & 72.755 & 3.309 & 14.332 \\
\hline $2-\mathrm{NI}$ & 0.08 & -39.949 & 56.771 & 130.884 & 72.305 & 58.580 & 1.752 & 19.135 \\
\hline 34-DNTul & 1.07 & -31.012 & 30.539 & 196.228 & 117.035 & 79.192 & 2.811 & 17.348 \\
\hline TNBP & 0.6 & -23.403 & 41.362 & 207.047 & 116.117 & 90.930 & 5.942 & 19.533 \\
\hline$m$-DNB & 0.01 & -26.105 & 31.540 & 175.830 & 103.652 & 72.178 & 3.952 & 16.800 \\
\hline$m-\mathrm{NBA}$ & 0.54 & -36.170 & 47.384 & 164.022 & 81.670 & 82.351 & 1.101 & 15.956 \\
\hline$m$-NTul & 1.07 & -33.993 & 22.297 & 171.182 & 114.510 & 56.672 & 2.034 & 10.779 \\
\hline NB & 0.85 & -32.983 & 25.334 & 150.292 & 100.930 & 49.362 & 1.785 & 10.861 \\
\hline $\mathrm{NBu}$ & 0.65 & -32.282 & 24.942 & 153.706 & 110.197 & 43.508 & 3.325 & 12.194 \\
\hline NEt & 1.10 & -31.882 & 25.038 & 110.544 & 67.801 & 42.743 & 2.412 & 15.773 \\
\hline $\mathrm{NMe}$ & 1.44 & -30.789 & 29.713 & 89.812 & 47.532 & 42.280 & 2.028 & 20.278 \\
\hline NPr & 0.65 & -32.066 & 25.315 & 132.338 & 89.159 & 43.179 & 3.084 & 13.782 \\
\hline$o-\mathrm{NBA}$ & 3.56 & -36.470 & 46.908 & 160.667 & 86.379 & 74.288 & 1.153 & 13.262 \\
\hline$o-\mathrm{NBP}$ & 2.828 & -38.030 & 61.879 & 157.862 & 93.308 & 64.584 & 1.169 & 15.022 \\
\hline$o$-NTul & 0.89 & -33.789 & 22.057 & 167.152 & 110.826 & 56.325 & 1.563 & 9.801 \\
\hline$p$-DNB & 0.0294 & -24.801 & 28.378 & 175.599 & 105.341 & 70.258 & 4.312 & 16.254 \\
\hline$p$-NBA & 3.25 & -40.397 & 50.522 & 163.927 & 85.016 & 78.911 & 1.151 & 17.195 \\
\hline$p$-NBP & 0.616 & -35.396 & 64.590 & 158.990 & 99.666 & 59.324 & 1.938 & 13.161 \\
\hline$p$-NTul & 2.14 & -34.575 & 21.802 & 171.128 & 114.284 & 56.844 & 2.176 & 11.221 \\
\hline Tetryl & 0.5 & -24.627 & 43.449 & 252.176 & 134.305 & 117.872 & 5.583 & 19.850 \\
\hline TNT & 0.48 & -21.892 & 35.556 & 216.994 & 125.037 & 91.956 & 5.674 & 17.784 \\
\hline LLM-105 & 2.000 & -38.131 & 44.554 & 196.467 & 108.710 & 87.756 & 3.206 & 19.441 \\
\hline Corr. & 1.0000 & -0.5745 & 0.2846 & -0.2131 & -0.3005 & -0.0576 & -0.5374 & -0.1135 \\
\hline
\end{tabular}

Note: $V_{\mathrm{S}, \min }, V_{\mathrm{S}, \max }, A_{S}, A_{S}^{+}, A_{S}^{-}, \bar{V}_{S}, \bar{V}_{S}^{+}, \bar{V}_{S}^{-}, v \sigma_{\text {tot }}^{2}, \Pi$ are in unit of kcal $/ \mathrm{mol}, \mathrm{kcal} / \mathrm{mol}, \AA^{2}, \AA^{2}, \AA^{2}, \mathrm{kcal} / \mathrm{mol}$, $\mathrm{kcal} / \mathrm{mol}, \mathrm{kcal} / \mathrm{mol},(\mathrm{kcal} / \mathrm{mol})^{2}$, $\mathrm{kcal} / \mathrm{mol}$, respectively. 
Table 6. 3 electrostatic potential parameters and 4 other parameters in relation to $L D_{50}^{\exp }$.

\begin{tabular}{|c|c|c|c|c|c|c|c|}
\hline & $\bar{V}_{S}^{-}$ & $v \sigma_{t o t}^{2}$ & $\Pi$ & $D \times 10^{7}$ & $N$ & $\log P_{\text {ow }}$ & $q_{\mathrm{NO}_{2}}$ \\
\hline $1-\mathrm{Cl}-2-\mathrm{NB}$ & -14.974 & 35.347 & 12.735 & 1.134 & 5 & 0.226 & -0.391 \\
\hline $1-\mathrm{Cl}-4-\mathrm{NB}$ & -12.733 & 30.160 & 10.796 & 1.475 & 5 & 0.584 & -0.402 \\
\hline 23-DNTul & -18.402 & 25.794 & 16.183 & 0.374 & 10 & -0.298 & -0.350 \\
\hline 24-DNBP & -12.995 & 25.237 & 13.901 & 0.498 & 12 & -0.424 & -0.396 \\
\hline 24-DNTul & -15.383 & 22.099 & 14.551 & 0.666 & 10 & 0.130 & -0.384 \\
\hline 25-DNTul & -14.189 & 17.889 & 13.460 & 0.880 & 10 & 0.602 & -0.379 \\
\hline 26-DNTul & -14.499 & 23.672 & 13.716 & 0.283 & 10 & 0.530 & -0.392 \\
\hline $2-\mathrm{NI}$ & -19.704 & 63.859 & 19.243 & -1.454 & 7 & -2.388 & -0.425 \\
\hline 34-DNTul & -18.674 & 26.914 & 17.382 & 0.395 & 10 & -0.717 & -0.359 \\
\hline TNBP & -11.414 & 27.261 & 15.731 & -1.565 & 17 & -0.476 & -0.344 \\
\hline$m$-DNB & -14.498 & 24.069 & 15.276 & 0.499 & 5 & 1.085 & -0.372 \\
\hline$m$-NBA & -13.632 & 69.061 & 14.822 & -1.317 & 6 & -1.539 & -0.417 \\
\hline$m$-NTul & -15.638 & 26.420 & 11.830 & 2.202 & 5 & -0.256 & -0.410 \\
\hline NB & -16.774 & 33.501 & 12.331 & 1.889 & 5 & -0.282 & -0.406 \\
\hline $\mathrm{NBu}$ & -19.138 & 19.114 & 12.773 & 2.003 & 5 & 0.580 & -0.325 \\
\hline NEt & -18.782 & 23.136 & 16.429 & 3.016 & 5 & -0.493 & -0.310 \\
\hline $\mathrm{NMe}$ & -18.386 & 26.452 & 19.293 & 3.675 & 5 & -0.894 & -0.303 \\
\hline $\mathrm{NPr}$ & -19.006 & 18.637 & 14.470 & 2.566 & 5 & 0.018 & -0.324 \\
\hline$o-\mathrm{NBA}$ & -12.927 & 58.162 & 13.068 & 0.203 & 6 & -1.375 & -0.496 \\
\hline$o-\mathrm{NBP}$ & -18.853 & 77.844 & 16.421 & -1.172 & 7 & -3.258 & -0.418 \\
\hline$o$-NTul & -14.646 & 27.471 & 10.999 & 1.921 & 5 & 0.306 & -0.425 \\
\hline$p$-DNB & -13.594 & 20.708 & 14.486 & 0.781 & 10 & 0.267 & -0.374 \\
\hline$p$-NBA & -16.135 & 90.226 & 16.680 & -1.889 & 6 & -3.110 & -0.462 \\
\hline$p$-NBP & -16.917 & 70.658 & 14.262 & -2.388 & 7 & -1.732 & -0.430 \\
\hline$p$-NTul & -16.009 & 27.983 & 12.228 & 2.217 & 5 & -0.449 & -0.417 \\
\hline Tetryl & -10.672 & 26.326 & 15.636 & -2.476 & 21 & -0.172 & -0.131 \\
\hline TNT & -10.793 & 20.144 & 14.33 & -0.678 & 15 & 0.888 & -0.355 \\
\hline LLM-105 & -16.906 & 45.439 & 18.068 & -1.276 & 16 & -3.885 & -0.3925 \\
\hline Corr. & -0.1735 & 0.5444 & 0.0630 & -0.03381 & & -0.6047 & -0.3860 \\
\hline
\end{tabular}

Note: $D$ is in unit of $\mathrm{cm}^{2} / \mathrm{s}$.

Table 7. The arrangement of correlation coefficients in descending order.

\begin{tabular}{rccccccccc}
\hline Para. & $\sigma_{-}^{2}$ & $\log P_{\mathrm{OW}}$ & $V_{\mathrm{S}, \min }$ & $\sigma_{\text {tot }}^{2}$ & $\varepsilon$ & $v \sigma_{\text {tot }}^{2}$ & $\bar{V}_{S}$ & $\delta^{2} V_{m}^{0.45}$ & $\gamma$ \\
\hline | corr. $\mid$ & 0.6814 & 0.6047 & 0.5745 & 0.5623 & 0.5473 & 0.5444 & 0.5374 & 0.5100 & 0.5060 \\
Para. & $\delta$ & $\mu$ & $q_{\mathrm{NO}_{2}}$ & $\sigma_{+}^{2}$ & $A_{S}^{+}$ & $E_{\text {coh }}$ & $\Delta_{\text {vap }} H^{\circ}$ & $V_{\mathrm{S}, \max }$ & $N V E$ \\
| corr. $\mid$ & 0.4566 & 0.4411 & 0.3860 & 0.3569 & 0.3005 & 0.2912 & 0.2911 & 0.2846 & 0.2346 \\
Para. & $\alpha(0)$ & $A_{S}$ & $V_{\mathrm{m}}$ & $\bar{V}_{S}^{-}$ & $\bar{V}_{S}^{+}$ & $\Pi$ & $A_{S}^{-}$ & $\nu$ & $D$ \\
| corr. $\mid$ & 0.2346 & 0.2131 & 0.1852 & 0.1735 & 0.1135 & 0.0630 & 0.0576 & 0.0439 & 0.03381 \\
\hline
\end{tabular}

The first columns in Table 3 and Table 5 are experimental values of $L D_{50}^{\exp }$, derived from the samples of rats (majority), mouses, rabbits, cats and dogs. The correlations between $L D_{50}^{\exp }$ and the parameters in Table 3 and Table 5 according to Equ. (15) were displayed in the last column in Table 3 to Table 6 , which were expressed in Table 7 in descending order, some electrostatic potentials based parameters, dielectric constants, surface tensions, solubility parameters, dipole moment make more contribution to the toxicity. The 9 parameters ranked high (correlation coefficient $\geq 0.3$ ) in Table 7 were considered in the screening process of genetic function approximation, $\sigma_{+}^{2}, \sigma_{-}^{2}, q_{\mathrm{NO}_{2}}, A_{S}^{+}, \bar{V}_{S}, v \sigma_{t o t}^{2}, \log P_{\text {OW }}$ were selected to build the relationship (Equ. (16)) with $L D_{50}^{\exp }$. 


$$
\begin{aligned}
& L D_{50}^{\text {cal }}=-0.012 X_{1}+0.024 X_{2}+38.855 X_{3}+0.164 X_{4}+0.028 X_{5}-0.227 X_{6}+1.017 \hat{R}\left(0.068-X_{7}\right)- \\
& 74.386(\hat{R}(X 3+0.494))^{\wedge} 2+346.992(\hat{R}(-0.364-X 3))^{\wedge} 2+0.0002(\hat{R}(117.057-X 5))^{\wedge} 2+17.241
\end{aligned}
$$

where $\mathrm{X}_{1}, \mathrm{X}_{2}, \mathrm{X}_{3}, \mathrm{X}_{4}, \mathrm{X}_{5}, \mathrm{X}_{6}$ and $\mathrm{X}_{7}$ represent $\sigma_{+}^{2}, \sigma_{-}^{2}, q_{\mathrm{NO}_{2}}, A_{S}^{+}, \bar{V}_{S}, v \sigma_{\text {tot }}^{2}, \mathrm{Log} P_{\mathrm{Ow}}$, respectively, $\hat{R}$ is ramp function [35], the square of correlation coefficient is 0.9724 . The experimental and predicted results were displayed in Table 8 and Figure 1.

\begin{tabular}{|c|c|c|c|c|c|c|c|c|c|}
\hline order & name & $L D_{50}^{\exp }$ & $L D_{50}^{\mathrm{cal}}$ & residual & order & name & $L D_{50}^{\exp }$ & $L D_{50}^{\mathrm{cal}}$ & residual \\
\hline 1 & 1-Cl-2-NB & 0.27 & 0.261 & -0.009 & 15 & $\mathrm{NBu}$ & $0.50-0.75$ & 0.850 & 0 \\
\hline 2 & 1-Cl-4-NB & 0.81 & 0.667 & -0.143 & 16 & NEt & 1.10 & 1.040 & -0.06 \\
\hline 3 & 23-DNTul & 1.12 & 0.992 & -0.128 & 17 & $\mathrm{NMe}$ & 1.44 & 1.480 & 0.04 \\
\hline 4 & 24-DNBP & 0.035 & 0.129 & 0.094 & 18 & NPr & $0.50-0.75$ & 0.558 & -0.092 \\
\hline 5 & 24-DNTul & 0.27 & 0.223 & -0.047 & 19 & $o-\mathrm{NBA}$ & 3.56 & 3.589 & 0.029 \\
\hline 6 & 25-DNTul & 0.71 & 0.573 & -0.137 & 20 & $o-\mathrm{NBP}$ & 2.828 & 2.769 & -0.059 \\
\hline 7 & 26-DNTul & 0.18 & 0.258 & 0.078 & 21 & $o-\mathrm{NTul}$ & 0.89 & 0.867 & -0.023 \\
\hline 8 & $2-\mathrm{NI}$ & 0.08 & 0.012 & -0.068 & 22 & $p$-DNB & 0.0294 & 0.135 & 0.1056 \\
\hline 9 & 34-DNTul & 1.07 & 1.088 & 0.018 & 23 & $p$-NBA & 3.25 & 3.216 & -0.034 \\
\hline 10 & TNBP & 0.6 & 0.507 & -0.093 & 24 & $p$-NBP & 0.616 & 0.630 & 0.014 \\
\hline 11 & $m$-DNB & 0.01 & 0.056 & 0.046 & 25 & $p$-NTul & 2.14 & 1.755 & -0.385 \\
\hline 12 & $m$-NBA & 0.54 & 0.729 & 0.189 & 26 & Tetryl & 0.5 & 0.498 & -0.002 \\
\hline 13 & $m$-NTul & 1.07 & 1.542 & 0.472 & 27 & TNT & 0.48 & 0.572 & 0.092 \\
\hline 14 & NB & 0.75-1.0 & 0.820 & -0.03 & 28 & LLM105 & 2.00 & 2.064 & 0.064 \\
\hline
\end{tabular}

Table 8. The comparison of predicted semi-lethal doses $\left(L D_{50}^{\mathrm{cal}}, \mathrm{g} / \mathrm{kg}\right)$ and experimental ones ( $\left.L D_{50}^{\exp }, \mathrm{g} / \mathrm{kg}\right)$.

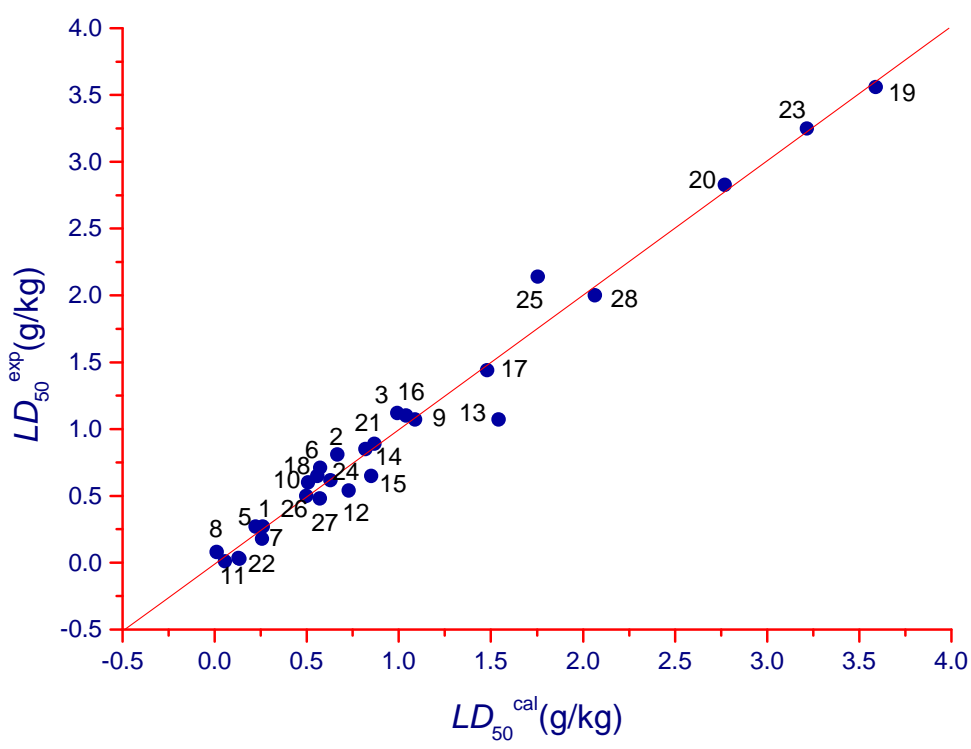

Figre 1. The relationship between experimental semi-lethal doses $\left(L D_{50}^{\exp }\right)$ and predicted ones $\left(L D_{50}^{\text {cal }}\right)$.

It is found from Figure 1 and Table 5 that the calculated semi-lethal doses $\left(L D_{50}^{\text {cal }}\right)$ according to Equ. (12) is in good agreement with experimental ones $\left(L D_{50}^{\exp }\right)$, and the squared-correlation coefficient reaches 0.9282 .

The screened parameters, the positive, negative and total variances-, $\sigma_{+}^{2}$ and $\sigma_{-}^{2}$ are effective index to extract the information contained in the electrostatic potential pattern over an entire molecular surface. These quantities indicate the molecule's capacity for noncovalent interactions through the regions of positive and negative potential on its surface. A variety of liquid, solid and solution phase 
properties that depend upon molecular interactions can be represented analytically in terms of these quantities plus the surface area. The product $v \sigma_{t o t}^{2}$, is especially important for representing properties that are related to the interactions of a molecule with others of its own kind [36]. $A_{S}^{+}$represents the positive superficial area of a molecular, which reflects the size of the molecule, and $\bar{V}_{S}$ represents the average molecular surface electrostatic potential.

At one time Mulliken charge of nitro group was applied to measure the sensitivity of nitro containing explosives, which reflects the detonation ability of self-oxidation reduction reactions [37], and was considered to evaluate the ability of self-oxidation reduction reactions in the organism, the more negative the $q_{\mathrm{NO}_{2}}$ is, the more difficultly the self-oxidation reduction reactions initiate. Solubility parameter $\delta$ reflects the extent of the similarity of the same type or different type molecules, the smaller the change $(\Delta \delta)$, the more similar the molecules, abiding by the rule of "like dissolves like" or "like seeks like" [38]. Log $P_{\mathrm{OW}}$ is the logarithm of oil/water partition coefficient for a compound, which reflects the relative size of water-solubility and fat-solubility. These multidimensional knowledges will help us to further understand the toxicity of the nitro containg compounds and reveal the toxicology and provide scintific basis for the design, the toxicity reduction and control of novel nitro-compounds.

\section{Conclusion}

Twenty seven parameters of twenty eight nitro-compounds were computed, which covered the areas of molecular surface properties, solubility, dielectric properties, polarizabilty and diffusibility. The correlation coefficients between the acute toxicity (semi-lethal doses) of 28 kinds nitro-compounds and these parameters were computed and sequenced in descending order. The results indicate that molecular surface electrostatic potentials based $\sigma_{-}^{2}, \sigma_{+}^{2}, \sigma_{t o t}^{2}, v \sigma_{t o t}^{2}, V_{\mathrm{S}, \min }, \bar{V}_{S}, A_{S}^{+}$, solubility related Log $P_{\mathrm{OW}}$, $\delta^{2} V_{m}^{0.45}, \delta$, dielectric constant $\varepsilon$, surface tension $\gamma$, dipole moment $\mu$, Mulliken charges related $q_{\mathrm{NO}_{2}}$ were arranged in front. Genetic function approximations, which model heridity, variation, natural selection and survival of the fittest, were performed to screen these parameters, $\sigma_{+}^{2}, \sigma_{-}^{2}, v \sigma_{t o t}^{2}, A_{S}^{+}, \bar{V}_{S}, q_{\mathrm{NO}_{2}}$, Log $P_{\text {Ow }}$ seven parameters were preserved to build the linear relation with toxicity $\left(L D_{50}\right)$. This work will not only lay a foundation for the toxicity of nitro-compounds, but also for other compounds, and it will provide basic references for the toxicology studies of compounds.

\section{Acknowledgement}

We will acknowledge the financial support of National Natural Science Foundation of China (11372289); Development Fund of CAEP: 2014-1-075. Some computations were carried out in Institute of Computational Science, CAEP.

\section{References}

1. V. Purohit and A. K. Basu, "Mutagenicity of nitroaromatic compounds," Chem. Res. Toxicol., vol. 13, pp. 673-692, 2000.

2. Y. Y. Liu, A. Y. H. Lu and R. A. Stearns, "In vivo covalent bingding of $\left[{ }^{14} \mathrm{C}\right]$ trinitrotoluene to proteins in the rat," Chem.Bio. Inter., vol. 82, pp.1-19, 1992.

3. G. Sabbioni, "Hemoglobin binding of nitroarenes and quantitative structure-activity relationships," Chem. Res. Toxicol., vol. 7, pp.267-274, 1994.

4. G. Sabbioni and O. Sepai, "Comparison of hemoglobin binding, mutagenicity and carcinogenicity of arylamines and nitroarenes," Chimica, vol. 49, pp.374-380, 1995.

5. N. Č. Aušra, M. Valè, ک̌. Jonas, M. Evaldas and Č. Narimantas, "Chemical aspects of cytotoxicity of nitroaromatic explosives: a review," CHEMIJA, vol. 17, pp.34-41, 2006.

6. K.T. Chung, C. A. Murdock, Y. Zhou, S. E. Stevens, Y. S. Li, C. Wei, S.Y. Fernando and M. W. Chou, "Effects of the nitro-group on the mutagenicity and toxicity of some benzamines," Enviro.Mol. Mutagen.,vol. 27, pp.67-74, 1996. 
7. A. Colombo, E. Benfenati and M.Karelson, "The proposal of architecture for chemical splitting to optimize QSAR models for aquatic toxicity," Chemosphere, vol. 72, pp. 772-780, 2008.

8. C. Hansch, P. P. Maloney, T. Fujita and R. M. Muir, "Correlation of biological activity of phenoxyacetic acids with hammett substituent constants and partition coefficients," Nature (London), vol. 194, pp.178-180, 1962.

9. C. Hansch and A. Kurup, "QSAR of chemical polarizability and nerve toxicity," J. Chem. Inf. Comput. Sci., vol. 43, pp.1647-1651, 2003.

10. A. R. Katritzky, M. Kuanar, S. Slavov and C. D. Hall, "Quantitative correlation of physical and chemical properties with chemical structure: utility for prediction," Chem. Rev., vol. 110, pp. 5714-5789, 2010.

11. S. Sild and M. Karelson, "A general QSPR treatment for dielectric constants of organic compounds," J. Chem. Inf. Comput. Sci., vol. 42, pp. 360-367, 2002.

12. M. Qasim, Y. Kholod, L. Gorb, D. Magers, P. Honea and J. Leszczynski, "Application of quantum-chemical approximations to environmental problems: Prediction of physical and chemical properties of TNT and related species," Chemosphere, vol. 69, pp. 1144-1150, 2007.

13. T. J. Sheldon, C. S. Adjiman and J. L. Cordiner, "Pure component properties from group contribution: Hydrogen-bond basicity, hydrogen-bond acidity, Hildebrand solubility parameter, macroscopic surface tension, dipole moment, refractive index and dielectric constant," Fluid Phase Equilibria, vol. 231, pp. 27-37, 2005.

14. P. Politzer, Y. Ma, P. Lane and M. C. Concha, "Computational prediction of standard gas, liquid, and solidphase heats of formation and heats of vaporization and sublimation," Inter. J. Quantum Chem., vol. 105, pp. 341-347,2005.

15. T. Singh and A. Kumar, "Static dielectric constant of room temperature ionic liquids: internal pressure and cohesive energy density approach," J. Phys. Chem. B, vol. 112, pp. 12968-12972, 2008.

16. S. J. Suresh and V. M. Naik,"Theory of dielectric constant of aqueous solutions," J. Chem. Phys., vol. 116, pp. 4212-4220, 2002.

17.V. Vasilyev, "Determination of the effective dielectric constant from the accurate solution of the poisson equation," J.Comput. Chem., vol. 23, pp. 1254-1265, 2002.

18. C. Caleman, P. J. van Maaren, M. Hong, J. S. Hub, L. T. Costa, and D.van der Spoel, "Force field benchmark of organic liquids: density, enthalpy of vaporization, heat capacities, surface tension, isothermal compressibility, volumetric expansion coefficient, and dielectric constant," J. Chem. Theory Comput., vol. 8, pp. 61-74, 2012.

19. C. Hansch, W. E. Steinmetz, A. J. Leo, S. B. Mekapati, A. Kurup and D.Hoekman, "On the role of polarizability in chemical-biological interactions," J. Chem. Inf. Comput. Sci. Vol. 43, pp. 120-125, 2003.

20. Y. Zhao and Z. Chai, "Nanotoxicology-safe use foundation of nanomaterials," Beijing: Science Press, pp.1-297, 2010.

21. P. Politzer and J. S. Murray, "Computational prediction of condensed phase properties from statistical characterization of molecular surface electrostatic potentials," Fluid Phase Equilibria, vol. 185, pp. 129-137, 2001.

22. J. S. Murray, T. Brinck and P. Politzer, "Relationships of molecular surface electrostatic potentials to some macroscopic properties," Chem. Phys., vol. 204, pp. 289-299, 1996.

23. P. Politzer, J. S. Murray and P. Flodmark, "Relationship between measured diffusion coefficients and calculated molecular surface properties," J. Phys. Chem., vol. 100, pp. 5538-5540, 1996.

24. P. Politzer, Y. Ma, P. Lane and M. C. Concha, "Computational prediction of standard gas, liquid, and solidphase heats of formation and heats of vaporization and sublimation," Inter. J .Quantum Chem., vol. 105, pp. 341-347, 2005.

25. T. Brinck; J. S. Murray and P. Politzer, "Octanol/water partition coefficients expressed in terms of solute molecular surface areas and electrostatic potentials," J. Org. Chem., vol. 58, pp. 7070-7073, 1993.

26. D .W. van Krevelen and K. te Nijenhius, "Properties of polymers," Bejing: Science Press, pp.190-319, 2010.

27. C. Hansch, W. E. Steinmetz, A. J. Leo, S. B. mekapati, A. Kurup and P. Hoekman, "On the role of polarizability in chemical- biological interactions," J. Chem. Inf. Comput. Sci., vol. 43, pp. 120-125, 2003.

28. T. J. Sheldon, C. S. Adjiman and T. L. Cordiner, "Pure component properties from group contribution, hydrogen-bond basicity, hydrogen-bond acidity, Hildebrand solubility parameters, macroscopic surface tension, dipole moment, refractive index and dielectric constant," Fluid Phase Equilibra, vol. 231, pp. 27-37, 2005.

29. A. D. Becke, "Density-functional thermochemistry. III. The role of exact exchange," J. Chem. Phys., vol. 98, pp. 5648-5652, 1993.

30. C. Lee, W. Yang and R. G. Parr, "Development of the Colle-Salvetti correlation-energy formula into a functional of the electron density," Phys. Rev. B, vol. 37, pp. 785-789, 1988.

31. T. Lu and F. Chen, "Multiwfn: A multifunctional wavefunction analyzer," J. Comput. Chem., vol. 33, pp. 580$592,2012$. 
32. G. Liu, L. Ma and S. Xiang, "Physical Properties Data Handbook in Chemical and chemical Industry (Organic)," Bejing: Science Press, pp.226, pp. 465, pp.499, 2012.

33. J. H. Holland, "Adaption in natural and atificial systems," The University of Michigan Press, $1^{\text {st }}$ ed, $2^{\text {nd }}$ ed. Cambridge, MA: NIT Press, 1992.

34. Y. Wen, "Toxicity data of Medicines and Chemicals," Tianjin China: Tianjin Science and Technology Press, pp.1-458,1989.

35. http://mathworld.wolfram.com/Ramp Function.html.

36. J. S. Murray and P. Politzer, "Statistical analysis of the molecular surface electrostatic potential: an approach to describing noncovalent interactions in condensed phases," J. Mol. Struct. (Theochem), vol. 425, pp. 107-114, 1998 .

37. C. Zhang, "Review of the establishment of nitro group charge method and its applications," J. Hazar. Mater. , vol. 161, pp. 21-28, 2009.

38. C. M. Hansen, "50 Year with solubility parameters-past and future," Progress in organic Coating, vol. 51, pp. 77-84, 2004.

\section{Appendix}

Table 9. The full names and Abbreviated names of the related nitro compounds.

\begin{tabular}{ll}
\hline Abbreviated name & Systematic nomenclature \\
\hline 1-Cl-2-NB & 1-chloro-2-nitrobenzene \\
1-Cl-4-NB & 1-chloro-4-nitrobenzene \\
23-DNTul & 2,3-dinitrotuluene \\
24-DNBP & 2,4-dinitrophenol \\
24-DNTul & 2,4-dinitrotuluene \\
25-DNTul & 2,5-dinitrotuluene \\
26-DNTul & 2,6-dinitrotuluene \\
2-NI & 2-nitroimidazole \\
$34-D N T u l$ & 3,4 -dinitrotuluene \\
TNBP & 2,4,6-trinitrophenol \\
m-DNB & 1,3 -dinitrobenzene \\
m-NBA & 3-nitrobenzenamine \\
m-NTul & 1-methyl-3-nitrobenzene \\
NB & nitrobenzene \\
NBu & 1-nitrobutane \\
NEt & nitroethane \\
NMe & nitromethane \\
NPr & 1-nitropropane \\
o-NBA & 2-nitrobenzenamine \\
o-NBP & 2-nitrophenol \\
o-NTul & 1-methyl-2-nitrobenzene \\
p-DNB & 1,4-dinitrobenzene \\
p-NBA & 4-nitrobenzenamine \\
p-NBP & 4-nitrophenol \\
p-NTul & 1-methyl-4-nitrobenzene \\
Tetryl & N-2,4,6-Tetranitro-N-methylaniline \\
TNT & $2,4,6$-trinitrotuluene \\
LLM-105 & 2,6-diamino-3,5-dinitropyrazine-1-oxide \\
\hline &
\end{tabular}

